\title{
Technè
}

La science au service de l'histoire de l'art et de la préservation des biens culturels

47 | 2019

Bernard Palissy : nouveaux regards sur la céramique française auX $\mathrm{XVI}{ }^{\mathrm{e}}$ et $\mathrm{XVII}{ }^{\mathrm{e}}$ siècles

\section{Sur les traces des producteurs de céramiques à glaçure plombifère et à décor moulé des règnes de Henri IV et de Louis XIII}

On the trail of producers of lead-glazed earthenware with moulded decoration during the reigns of Henri IV and Louis XIII

\section{Jessica Denis-Dupuis}

\section{OpenEdition Journals}

Édition électronique

URL : http://journals.openedition.org/techne/1512

DOI : 10.4000/techne.1512

ISSN : 2534-5168

\section{Éditeur}

C2RMF

\section{Édition imprimée}

Date de publication : 1 juin 2019

Pagination : 62-71

ISBN : 978-2-11-152830-7

ISSN : $1254-7867$

\section{Référence électronique}

Jessica Denis-Dupuis, "Sur les traces des producteurs de céramiques à glaçure plombifère et à décor moulé des règnes de Henri IV et de Louis XIII », Technè [En ligne], 47 | 2019, mis en ligne le 01 juin 2020, consulté le 23 juillet 2020. URL : http://journals.openedition.org/techne/1512 ; DOI : https:// doi.org/10.4000/techne.1512

\section{(c) (i) (9)}

La revue Technè. La science au service de l'histoire de l'art et de la préservation des biens culturels est mise à disposition selon les termes de la Licence Creative Commons Attribution - Pas d'Utilisation Commerciale - Pas de Modification 4.0 International. 


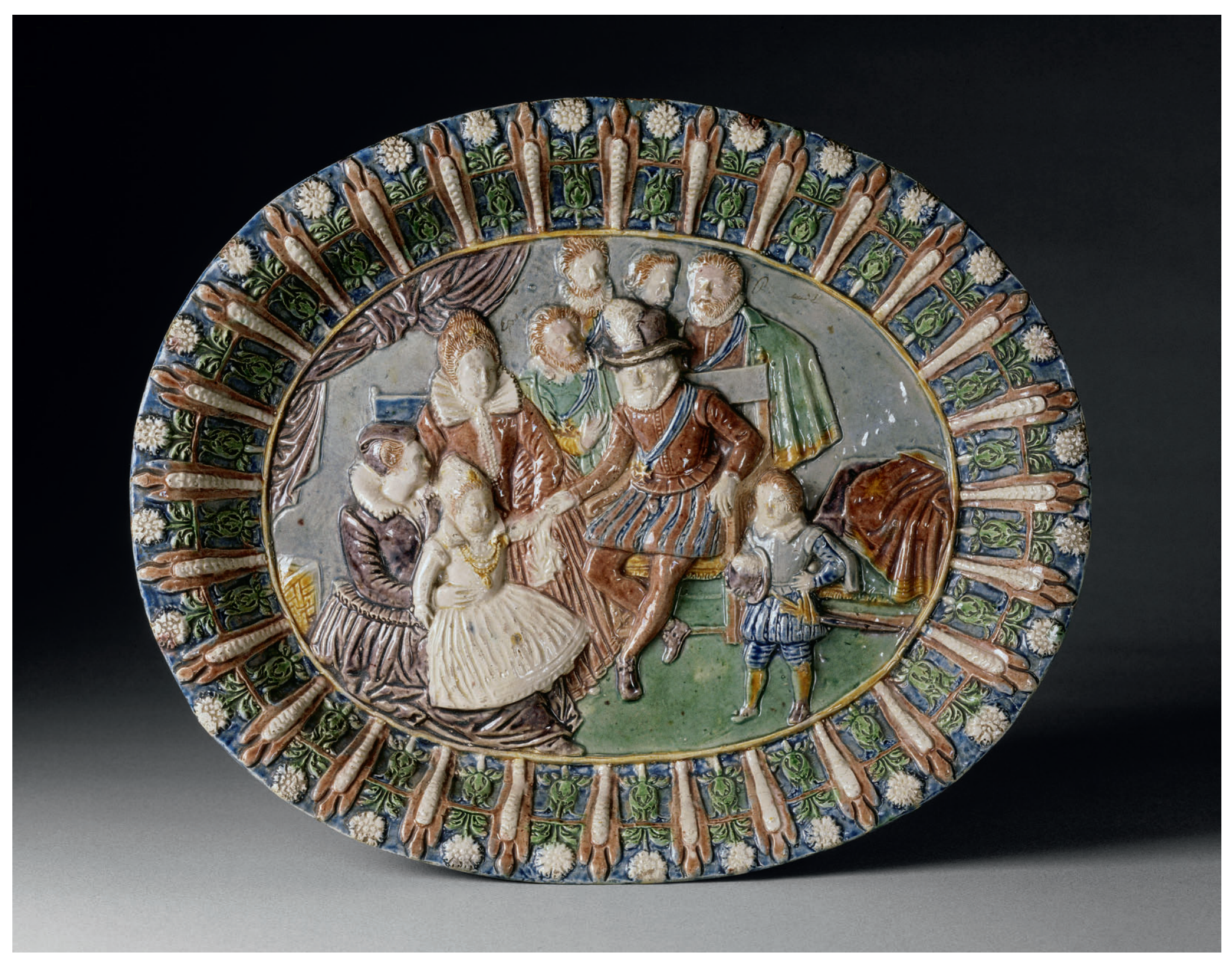

Fig. 1. Paris (?), première moitié du Xvil ${ }^{\mathrm{e}}$ siècle, Plat ovale, Henri IV et sa famille,

terre cuite à glaçure plombifère (L. 33,2 cm, 1. 27,3 cm, H. $6 \mathrm{~cm}$ ), Paris, musée du Louvre,

département des Objets d'art, OA 1351. (C) RMN-Grand Palais (musée du Louvre)/Daniel Arnaudet. 
Jessica Denis-Dupuis

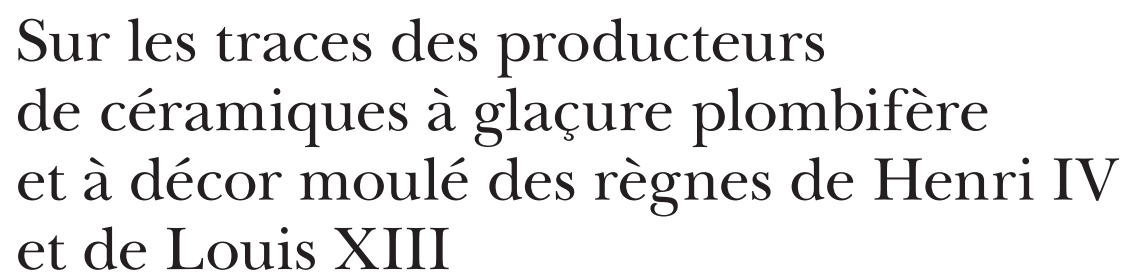

On the trail of producers of lead-glazed earthenware with moulded decoration during the reigns of Henri IV and Louis XIII

Résumé. Après une première avancée permettant de faire la distinction entre céramiques palisséennes et céramiques postpalisséennes, les recherches menées dans les archives permettent aujourd'hui de réviser l'histoire légendaire des céramiques postpalisséennes et de mieux les situer dans une époque et dans un contexte de production. Alors que l'on pensait l'ensemble de ces objets issu de la cité royale de Fontainebleau et de l'atelier dit "d'Avon ", la mise au jour de documents d'archives inédits démontre l'importance de Paris dans la fabrication et la commercialisation de pièces céramiques dans la première moitié du XVII ${ }^{e}$ siècle. Dans le faubourg Saint-Germain-des-Prés, Daniel et Aggée Cattier, deux frères "émailleurs en terre ", fabriquent et vendent des "terres émaillées » issues de moules en plâtre. Ils appartiennent à un large réseau d'émailleurs en terre dont on recense des membres à Fontainebleau, à Rouen et jusqu'en Angleterre. Dans le même temps, Antoine Clarissy est à la tête d'une importante fabrique de céramiques dans la capitale qui produit des "terres sigillées » à destination d'une clientèle aisée et prestigieuse, ainsi qu'en témoignent les inventaires après décès.

Mots-clés. Palissy, céramique post-palisséenne, glaçure plombifère, terre sigillée, Paris, Saint-Germain-des-Prés, Fontainebleau, Avon, émailleurs en terre, Chipault, Berthélémy, Cattier, Séguier, Clarissy.
Abstract. Having initially made enough headway to distinguish between Palissian ceramics and post-Palissian ceramics, research undertaken in the archives today enables us to revise the habitual story of post-Palissian ceramics and to better situate them in a period and context of production. Whereas it was previously thought that all of these objects came from the royal city of Fontainebleau and the "Avon" pottery workshop, the updating of hitherto-unpublished archive documents has shown the importance of Paris in the manufacture and sale of ceramic pieces in the first half of the 17th century. In the Faubourg Saint-Germain-des-Prés, two brothers, Daniel and Aggée Cattier, "earthenware glazers" by trade, made and sold "glazed earthenware" pieces cast in plaster moulds. They belonged to a wide network of potters and glazers whose members were active in Fontainebleau, Rouen and England. In this same period, Antoine Clarissy ran a large pottery factory in the French capital that produced "terres sigillées", as attested by the inventories of a wealthy, prestigious clientele.

Keywords. Palissy, post-Palissian ceramics, lead glaze, terra sigillata, Paris, Saint-Germain-des-Prés, Fontainebleau, Avon, earthenware glazers, Chipault, Berthélémy, Cattier, Séguier, Clarissy.

\section{La fin de la légende palisséenne}

Les céramiques à glaçure plombifère et à décor moulé en relief, que l'on nomme encore parfois «suites de Palissy ", sont aujourd'hui définitivement libérées des derniers reliquats de légende palisséenne. Ce résultat est le fruit de recherches ayant débuté il y a plusieurs décennies avec les fouilles archéologiques menées sur le site des Tuileries dans le cadre du projet du Grand Louvre entre 1984 et 1987. À cette occasion, les archéologues mettent en effet au jour un atelier de production céramique identifié comme étant celui de Bernard Palissy et cette découverte met à disposition des chercheurs un grand nombre de données nouvelles ${ }^{1}$. Ces données permettent d'une part de distinguer clairement l'œuvre céramique issue de l'atelier de Bernard Palissy parmi l'immense ensemble existant de statuettes et pièces de vaisselle d'apparat en céramique à glaçure plombifère et à décor moulé. D'autre part, elles permettent de faire le point sur la part d'héritage et d'influence aussi bien technique que stylistique de Palissy sur l'ensemble de cette production que l'on attribuait jusqu'ici à de supposés « suiveurs » ou « continuateurs » qui n'auraient fait que reproduire pendant plusieurs décennies des modèles créés par le maître potier. Le compterendu des fouilles démontre que la durée d'utilisation de cet 
atelier se situe entre 1565-1566 et 1584-1590, mais pas au-delà. En outre, bien qu'un grand nombre de fragments mis au jour se rapporte à des pièces de vaisselle d'apparat dont certains présentent des motifs figurés ou décoratifs, aucun d'entre eux ne permet réellement d'être mis en rapport avec la production post-palisséenne - excepté peut-être les quelques fragments relatifs au bassin dit «du Déluge » (voir encadré du même auteur dans ce volume). Par conséquent, ces productions dites «post-palisséennes » ne peuvent être considérées stylistiquement comme un héritage palisséen, ce que confirment par ailleurs les décors en relief de certaines de ces céramiques dont les datations sont nécessairement postérieures, comme celles illustrant Henri IV et sa famille (fig. 1) ou Louis XIII.

À la lumière des analyses des fragments palisséens, l'héritage technique de l'activité de Palissy doit lui aussi être relativisé : les analyses physico-chimiques comparatives menées sur les vestiges mis au jour démontrent que, contrairement aux œuvres de ses «suiveurs" (fig. 2), la dizaine de pièces de vaisselle palisséenne à décor de rustiques figulines aujourd'hui conservée (fig. 3) ${ }^{2}$ est le résultat de la mise en œuvre de techniques complexes utilisant une grande diversité de matériaux. Bernard Palissy parvient en effet à mêler des glaçures plombifères avec des glaçures riches en plomb opacifiées à l'oxyde d'étain ${ }^{3}$. Il joue ainsi sur les épaisseurs et les transparences, tel un illusionniste souhaitant rivaliser avec la nature. Pour réaliser la vaisselle de terre, il emprunte aux domaines de l'orfèvrerie ou de la médaille différents modèles en relief qui, une fois transposés dans un moule, sont combinés pour donner naissance à des créations originales. Il est donc un véritable chercheur et, à ce titre, son atelier, où l'on expérimente et met au point des techniques très poussées de l'art de la terre, a pu être qualifié d' " atelier d'art et d'essai ${ }^{4}$ ».

Techniquement, les œuvres produites sous les règnes de Henri IV et de Louis XIII n'ont rien de comparable. Elles ne font que reproduire une recette beaucoup plus simple dont les couleurs sont obtenues à l'aide d'une glaçure plombifère transparente colorée par des oxydes métalliques ${ }^{5}$. Ceux que l'on a nommés les «suiveurs » de Bernard Palissy, qui reprennent la technique du moulage pour réaliser de la vaisselle d'apparat, diversifient également la source de leurs décors en relief (qui était exclusivement des modèles d'orfèvrerie pour Palissy). Ils n'hésitent pas à puiser parmi les arts graphiques et en particulier la gravure : ils ont donc acquis la capacité de transposer en relief des modèles en deux dimensions et allient ainsi les compétences du céramiste et celles du sculpteur. Par ailleurs, les quantités de vaisselle produite en série à partir d'un même moule démontrent également une démarche bien différente de celle de Palissy : il n'est plus question d'expérimentations ni de recherches, mais d'une production sérielle à visée commerciale.

\section{Vers de nouvelles hypothèses}

Même si cette distinction est aujourd'hui clairement établie, il n'en demeure pas moins qu'après avoir été pendant près de deux siècles interprétées, étudiées, acquises et collectionnées comme des œuvres du maître des Tuileries ou héritières de son art, toute rupture définitive avec Bernard Palissy est encore parfois difficile à consommer. En effet, le vocabulaire employé pour qualifier ce type de céramiques du règne de Henri IV ou de Louis XIII dites «suites de Palissy » ou bien encore « post-palisséennes », fait toujours référence au maître potier $\mathrm{du} \mathrm{XvI}{ }^{\mathrm{e}}$ siècle. Il convient pourtant de changer définitivement de terminologie, cette dénomination n'ayant plus de raison d'être, mais pour ce faire, encore faut-il pouvoir proposer une alternative plus rigoureuse et pertinente.

Une fois l'hypothèse palisséenne définitivement écartée, se pose la question de l'interprétation des vestiges se rapportant à ces céramiques, découverts non loin d'un four de potier dans d'autres centres de production français tels la Normandie ou la Saintonge ${ }^{6}$. Faut-il alors accorder à ces localités l'ensemble de la production dont un premier recensement non exhaustif excède déjà le millier de pièces dans les seules collections publiques ${ }^{7}$ ? L'étude des comptes-rendus archéologiques laisse plutôt penser, au vu du faible nombre de ces fragments mais aussi des différences stylistiques ou techniques qu'ils présentent, qu'il s'agit surtout de tentatives locales de reproduction d'objets nés et importés d'un autre foyer de production principal qui aurait, quant à lui, développé une production sérielle et dont la diffusion aurait été largement faite dans le pays. En ce qui concerne le potentiel foyer dit « d'Avon » ou de Fontainebleau auquel nombre de ces céramiques ont été attribuées (fig. 4), rappelons qu'aucune preuve n'a pu être apportée par l'archéologie et que le principal document d'archive invoqué par Félix Herbet n'a malheureusement, jusqu'à ce jour, pas encore pu être localisé et authentifié ${ }^{8}$.

D'un point de vue historique, les recherches concernant ces céramiques à glaçure plombifère de la fin du Xvi $\mathrm{I}^{\mathrm{e}}$ siècle et de la première moitié du XVII ${ }^{\mathrm{e}}$ siècle se trouvent au point mort depuis plus d'un siècle, faute de nouvelle hypothèse à explorer. Bien que probablement français, ce foyer principal de fabrication pouvait en effet être localisé en n'importe quel lieu du royaume. Une nouvelle hypothèse géographique était donc nécessaire afin de relancer les recherches en archives. L'élaboration de cette nouvelle hypothèse a été rendue possible grâce à l'étude effectuée par le géologue Jean-Claude Plaziat sur les plats à décor de rustiques figulines. En effet, ce dernier a démontré que les moulages de fossiles de coquillages que l'on retrouve en grand nombre sur les céramiques rustiques post-palisséennes ne sont jamais présents sur les œuvres authentiques de Bernard Palissy. Ces fossiles sont des fossiles marins du Lutétien moyen et supérieur, du Cuisien et du Sparnacien (Yprésien) du Bassin parisien, provenant de gisements du Nord et de l'Est de Paris s'étendant du Vexin à la Vallée de la Marne ${ }^{9}$. Il est probable que ces fossiles se soient trouvés mêlés au sable utilisé par les céramistes à l'origine de 


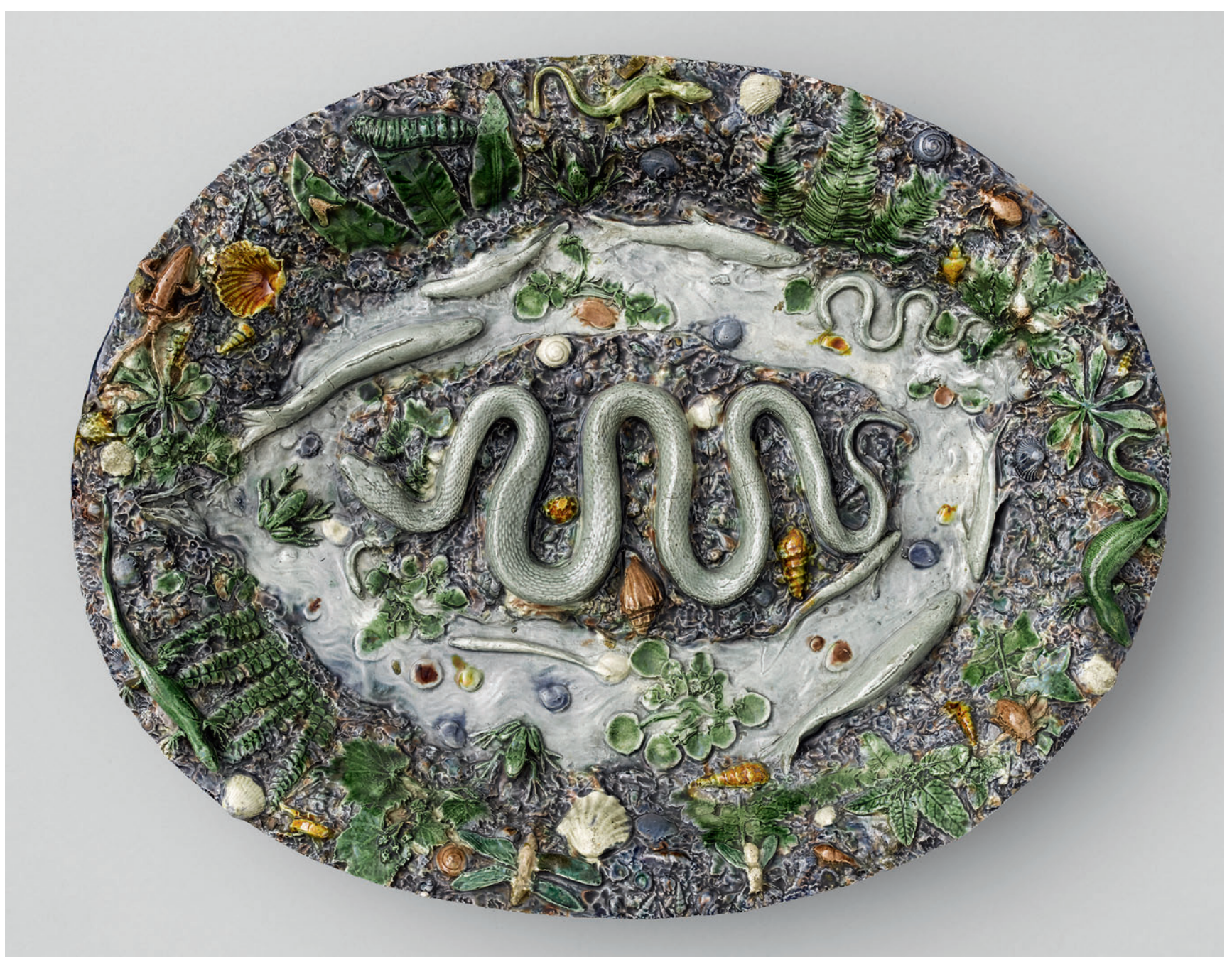

Fig. 2. Paris (?), première moitié du $\mathrm{XVII}^{\mathrm{e}}$ siècle, Plat ovale à décor de rustiques figulines, terre cuite à glaçure plombifère (L. $51 \mathrm{~cm}, 1.40 \mathrm{~cm}, \mathrm{H} .7 \mathrm{~cm}$ ), Paris, musée du Louvre, département des Objets d'art, OA 1358. @ RMN-Grand Palais (musée du Louvre)/Jean-Gilles Berizzi.

ces productions. Le lieu d'approvisionnement en sable étant sans doute situé non loin du lieu de fabrication, l'hypothèse d'un centre de fabrication situé au cœur ou à proximité de la capitale a constitué le point de départ des recherches menées dans les archives.

Cette hypothèse se devait d'autant plus d'être explorée que les sources d'archives parisiennes n'ont jusqu'ici jamais été étudiées en ce qui concerne la céramique de la fin du $\mathrm{XVI}^{\mathrm{e}}$ siècle et de la première moitié $\mathrm{du} \mathrm{XVII}^{\mathrm{e}}$ siècle. Les recherches menées ont ainsi permis de réévaluer nombre de croyances anciennes et de révéler que les «suiveurs " ou « continuateurs » de Bernard Palissy dont les noms étaient jusqu'ici rattachés à l'atelier dit «d'Avon » près de Fontainebleau ont en fait des attaches bien plus fortes dans la capitale.

Depuis l'article publié en 1897 par Félix Herbet sur les « émailleurs en terre »de Fontainebleau ${ }^{10}$, l'histoire légendaire reliant directement l'atelier de Bernard Palissy aux Tuileries à celui d'Antoine Clarissy à Avon, en passant par les « émailleurs en terre » de Fontainebleau, a été maintes et maintes fois reprise et répétée $\mathrm{e}^{11}$. Quelques hypothèses élaborées à partir d'éléments découverts dans les archives se sont ainsi vues transformées au fil du temps en faits, sans que ceuxci n'aient toutefois jamais pu être vérifiés par l'archéologie ou de nouveaux documents d'archives. C'est ainsi que l'on aura pu lire que Bernard Palissy eut pour associé Jean Chipault, orfèvre et émailleur, dont le fils du même nom aurait été à l'origine de l'atelier de Fontainebleau aux côtés des «émailleurs en terre » Claude Berthélémy et Claude Beaulat, dont la veuve de ce dernier se serait associée à Antoine Clarissy à Avon. Les recherches menées dans les archives n'ont à ce jour pas permis d'attester de filiation entre ces divers fabricants de céramique et tendent même à démontrer que la réalité est beaucoup plus complexe et fait intervenir un bien plus grand 
nombre d'acteurs. Grâce aux actes d'archives pour la plupart inédits mis au jour dans le cadre de cette étude ${ }^{12}$, il n'est désormais plus question de continuer d'invoquer cette légende.

\section{Les « émailleurs en terre » du faubourg Saint-Germain-des-Prés}

Jean Héroard, médecin du jeune dauphin et futur Louis XIII, rapporte dans son Journal l'existence, dans les premières années du XVII ${ }^{\mathrm{e}}$ siècle, d'une poterie située près du château de Fontainebleau où se rend le jeune Louis pour acquérir de petites statuettes de terre qu'il considère comme ses jouets (fig. 4) ${ }^{13}$. Lorsque la naturalisation de Claude Berthélémy, " émailleur en terre » originaire de Lorraine, par lettres patentes du roi Henri IV datant de 1602, est découverte parmi les archives de Fontainebleau ${ }^{14}$, on suppose que les fabricants de statuettes de la poterie du château évoqués par le médecin royal et les «émailleurs en terre » de Fontainebleau dont Félix Herbet révèle les noms sont les mêmes personnes. Claude Berthélémy ayant épousé en premières noces une certaine Suzanne Chipault, il en est déduit, mais sans plus de preuves, que cette dernière est apparentée à Jean Chipault, " émailleur en terre » rattaché à la Maison du Roi de 1599 à $1611^{15}$. Les recherches menées au sein du Minutier central des

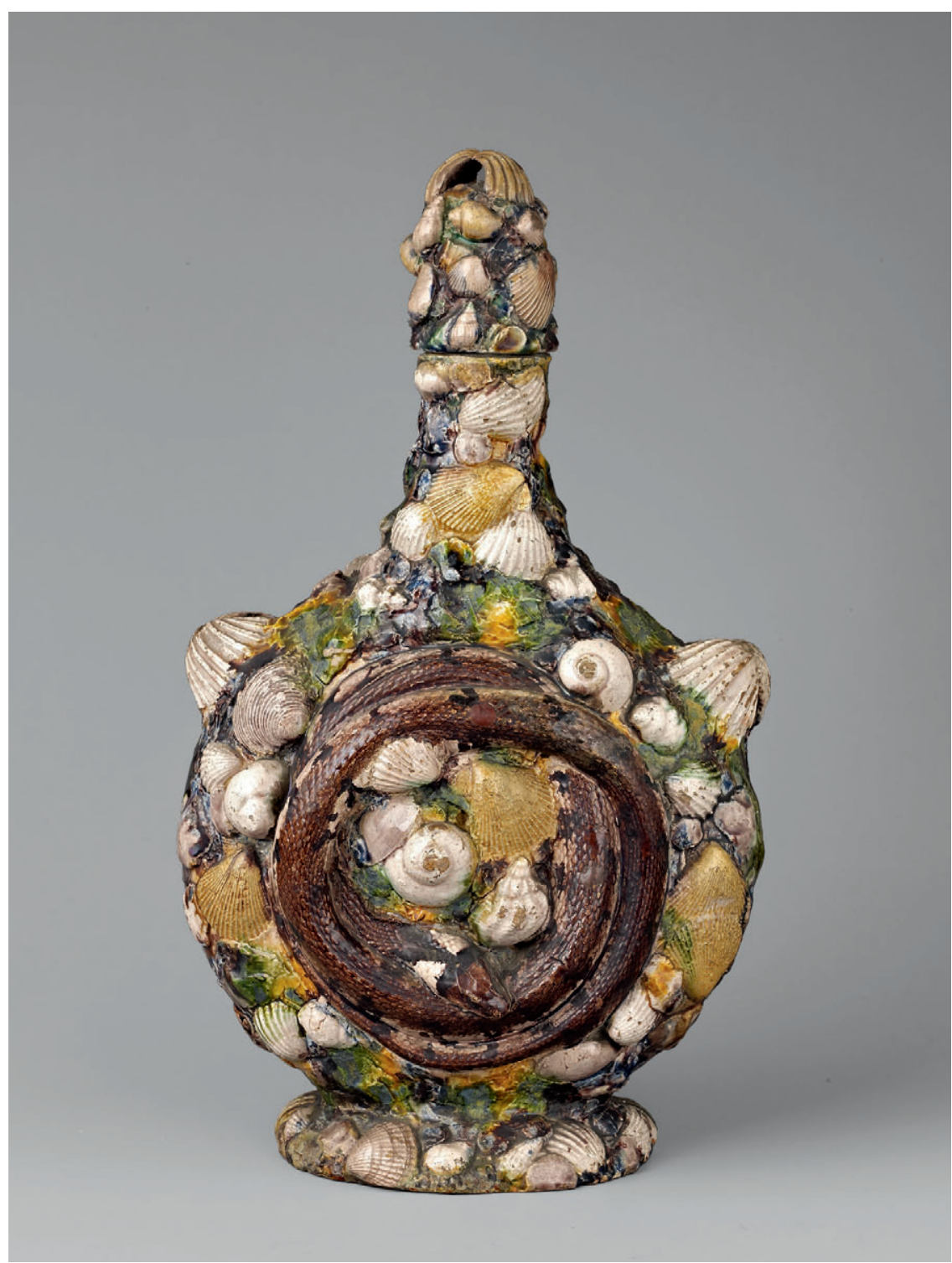

Fig. 3. Bernard Palissy, Saintes (?), vers 1555-1565, Gourde de pèlerin à décor de rustiques figulines, terre cuite glaçurée (H. 31 cm, L. 19 cm), New York, Metropolitan Museum, Robert Lehman Collection, 1975.1.1620. Met Museum CC License/Public Domain. notaires de Paris n'ont pas permis de vérifier cette parenté, mais ont en revanche révélé la parenté entre Suzanne Chipault et deux autres «émailleurs en terre » du même nom : Pierre et Benjamin Chipault, tous deux installés dans le faubourg Saint-Germain-des-Prés dans les dernières décennies du $\mathrm{XVI}^{\mathrm{e}}$ siècle $^{16}$. S'il demeure possible que ces derniers soient apparentés à l'émailleur de la Maison du Roi Jean Chipault, il ne s'agit pour l'heure que d'une hypothèse, de même que l'origine présumée saintongeaise de la famille Chipault. Par conséquent, en l'état actuel de nos connaissances, toute filiation entre Bernard Palissy et les « émailleurs en terre » de Fontainebleau ou du faubourg Saint-Germain-des-Prés de Paris n'est que pure fiction.

Les recherches menées dans les archives parisiennes ont également révélé la présence de deux frères « émailleurs en terre » nommés Daniel et Aggée Cattier installés dans le faubourg Saint-Germain-des-Prés ${ }^{17}$. Originaires de Picardie et fils d'un tourneur sur bois, tous deux sont cousins de Suzanne Chipault, la première épouse de Claude Berthélémy. Il est fort probable qu'ils aient appris leur métier auprès de Pierre ou Benjamin Chipault à Saint-Germain-des-Prés et que ce soit le décès de l'un de ces deux émailleurs qui motive l'installation de leur propre atelier de fabrication de céramiques dans ce même faubourg de Paris. Ils acquièrent en effet en 1602 un terrain avec une masure située rue des Fossés entre les portes de Bussy et de Nesle pour 750 livres tournois. Dès 1607, ils y font bâtir une seconde maison du côté de la rue de Seine, de telle sorte que chaque frère dispose d'une maison, la première donnant sur la rue des Fossés et la seconde sur la rue de Seine. Entre les deux s'étend un jardin dans lequel se trouve leur four d'émailleurs ainsi que des 
appentis. Daniel Cattier est propriétaire du four, mais dans la mesure où chacune des deux maisons comprend une boutique et où Aggée Cattier possède une loge à la foire Saint-Germain ${ }^{18}$, il est très probable que tous deux exercent ensemble. Daniel Cattier acquiert une seconde loge à la foire Saint-Germain en 1617, ce qui représente pour ces frères émailleurs un double étal où exposer leurs marchandises de terre. Le fait qu'aucun de leurs enfants ne poursuive l'activité professionnelle familiale et que leurs filles épousent des peintres, orfèvres ou marchands bourgeois de Paris témoigne d'une forte implication de ces « émailleurs en terre » dans un milieu artistique et marchand, ainsi que de leur ascension sociale. On recense en effet dans leur entourage un grand nombre d'artistes et d'artisans importants du domaine du luxe, à l'instar des horlogers de la famille Greban, des peintres de la famille Elle, des orfèvres de la famille Toutin ou encore de sculpteurs tel Pierre Collot. Au décès de Daniel Cattier, l'estimation totale de ses biens immobiliers représente la somme de 47000 livres tournois, somme exceptionnelle pour un «émailleur en terre » ayant débuté ses activités en 1602 par l'achat d'un terrain et d'une masure en communauté avec son frère. À ce patrimoine immobilier constitué ex nihilo, il faut encore ajouter l'importance des biens mobiliers et de l'argent comptant dont témoigne l'inventaire après décès de Daniel Cattier en $1642^{19}$. Cet inventaire recèle en outre de très précieuses informations sur l'atelier de « terres émaillées » que possède l'émailleur. Bien que l'absence de matières premières telles que la terre ou l'émail tendrait à indiquer qu'à cette date, Daniel Cattier n'a peut-être pas luimême fabriqué cette vaisselle, son activité marchande ne fait aucun doute : les céramiques présentes alors dans la maison sont au nombre de 21245 pièces et valent en moyenne entre 20 sols et 50 sols la douzaine. Ces dernières ne sont malheureusement pas décrites avec précision, mais les typologies mentionnées par le notaire sont intéressantes : il s'agit de plats, vases, pots à eau, aiguières, écuelles, saladiers, salières, flambeaux, bassins à barbe, bassins, tableaux en basse taille, pièces de poêle et "figures » de chevaux, lions et éléphants qui sont de petites statuettes animalières. La quantité importante de 500 moules en plâtre ayant été dénombrée dans la maison de Daniel Cattier, il s'agit bien de céramique moulée et non tournée. Par conséquent, la production est variée et donne lieu à une fabrication de pièces en série. Mis à part les plats à barbe, dont aucun exemplaire en terre cuite à glaçure plombifère et à décor moulé n’a été recensé jusqu'à présent, le reste des typologies de céramiques mentionnées dans l'inventaire pourrait correspondre aux céramiques post-palisséennes. Toutefois, l'inventaire après décès ne mentionne jamais de décor de rustiques figulines ou de décor figuré, ornemental ou ajouré, tout au plus précise-t-on qu'une partie de ces pièces est «façon d'agathe », ce que l'on pourrait interpréter comme étant des «terres mêlées ${ }^{20}$ ». L'inventaire atteste quoi qu'il en soit que ces « émailleurs en terre » sont bien à l'origine de la fabrication de statuettes à glaçure plombifère.
Cette famille d'« émailleurs en terre » parisiens et bellifontains est en outre beaucoup plus large qu'on aurait pu le penser et ses ramifications sont très étendues : une branche de la famille Berthélémy fabrique de la céramique à Rouen dans la première moitié $d u X{ } I^{\mathrm{e}}$ siècle. La présence de Jean Berthélémy, "émailleur en terre ", y est en effet attestée dès $1608^{21}$. Ainsi que le révèle un acte du tabellionage de Rouen daté de $1621^{22}$, ce dernier est en affaires avec Claude Beaulat, l'« émailleur en terre » de Fontainebleau. Par conséquent, la parenté avec les Berthélémy établis dans la cité royale de Fontainebleau paraît extrêmement probable. Cet acte d'archives révèle également qu'à cette date, Claude Beaulat se trouve à Londres en qualité de marchand. Son épouse nommée Anne Berthélémy pourrait d'ailleurs bien être la fille de Jean Berthélémy de Rouen. Claude Beaulat ${ }^{23}$ semble s'être spécialisé dans le commerce et la diffusion sur le territoire national et à l'étranger des céramiques issues des moules des « émailleurs en terre ». En effet, il conclut en 1615 un contrat de transport avec un marchand de Fontainebleau chargé de transporter « trois caisses plaines de marchandise de son estat d'esmailleur » dans la ville de Lyon et s'il est présent à Londres de 1621 à 1623, c'est en qualité de marchand et d'intermédiaire de l'"émailleur en terre "rouennais, lequel lui confie ses productions qu'il est chargé de vendre à la clientèle londonienne. Ainsi, bien que nous ne disposions à ce jour que de peu d'informations sur ses activités, Claude Beaulat étant un céramiste mentionné parmi les artistes appartenant à la Maison du Roi pour les années 1631 à $1637^{24}$, il est probablement le plus important de tous ces « émailleurs en terre » durant cette période. Ses activités et ses pérégrinations démontrent l'existence de connexions entre divers centres de production et permettent de suivre certains chemins de commercialisation empruntés par les céramiques parisiennes, bellifontaines et rouennaises de la première moitié du XVII ${ }^{\mathrm{e}}$ siècle.

\section{Antoine Clarissy et les mystérieuses «terres sigillées »}

Selon le père Dan, qui publie en 1642 son ouvrage intitulé le Trésor des merveilles de la Maison royale de Fontainebleau, Antoine Clarissy fabrique des ouvrages de terre sigillée et établit sa verrerie royale au domaine du Monceau situé au bout du parc du château de Fontainebleau dépendant de la paroisse d'Avon ${ }^{25}$. L'origine du foyer dit «d'Avon » et l'idée selon laquelle Antoine Clarissy serait l'un des continuateurs de Bernard Palissy dans l'art céramique proviennent de cette unique mention. Toutefois, s'il atteste la double activité céramique et verrière d'Antoine Clarissy, le père Dan ne localise pas clairement l'atelier de fabrication de terres sigillées d'Antoine Clarissy dans la Verrerie royale du Monceau et aucun document relatif à cette verrerie n'est pour l'heure venu confirmer qu'il s'y fabriquât un jour des pièces de céramique. En revanche, celui qui porte le titre «d'ouvrier du roi en terre 
sigillée » est bien à la tête d'un atelier de céramique situé non pas à Fontainebleau, mais en plein cœur de la capitale.

Antoine Clarissy ${ }^{26}$ arrive à Paris en 1612, appelé par le roi depuis la ville de Marseille. Un acte issu du Minutier central de Paris datant du 4 janvier 1612 témoigne de l'installation d'Antoine Clarissy dans la capitale. Il s'agit d'un bail en faveur de Clarissy passé au nom du roi par Jehan de Fourcy, intendant des bâtiments de Sa Majesté avec les religieux des Feuillants du faubourg Saint-Honorée ${ }^{27}$. L'ouvrier céramiste qualifié de potier et maître en terre sigillée qui s'attribue par la suite le qualificatif d'ouvrier du roi n'a pas usurpé son titre. Si le roi le loge gracieusement dans une maison dépendant du couvent des Feuillants, c'est en échange de quatre mois de travail au service de la monarchie et des bâtiments royaux et dans l'attente de la vacance d'un logement dans la Grande Galerie du Louvre, où le roi loge les artistes travaillant à son service. Antoine Clarissy n'obtient finalement jamais son logement dans la Grande Galerie du Louvre, mais reçoit à la place, probablement dès 1616, un logement dans le Palais des Tuileries. L'attribution d'un logement dans le Palais des Tuileries n'en est pas moins prestigieuse puisque les artistes qui y sont logés jouissent des mêmes privilèges que leurs collègues du Louvre ${ }^{28}$. On recense parmi les voisins d'Antoine Clarissy au Palais des Tuileries les sculpteurs Francesco Bordoni dès 1616, Philippe de Buyster en 1632 puis François Anguier, les peintres Jacques d'Hoey et Jean Nocret et des orfèvres tels François Roberday ou après 1639, Claude Ballin ${ }^{29}$. Dans la mesure où un grand nombre des artistes et artisans bénéficiant de brevets royaux et dépendant de la Surintendance des Bâtiments du Roi se voient à cette époque également gratifiés par le roi de magasin, boutique, cour ou terrain dépendants du Palais ${ }^{30}$, il est probable qu'Antoine Clarissy ait lui aussi bénéficié d'une place située dans le jardin des Tuileries ou proche de celui-ci pour y loger son atelier de terres sigillées. Clarissy est d'ailleurs l'un des seuls artistes bénéficiant d'une concession de neuf lignes d'eau ${ }^{31}$ provenant des eaux de la pompe du roi ${ }^{32}$. Dans la mesure où le travail de l'argile requiert un usage abondant en eau pour être purifiée avant façonnage, moulage et cuisson par le céramiste, ces neuf lignes d'eau constituent un indice supplémentaire propre à localiser l'atelier de Clarissy dans l'enceinte du Palais des Tuileries.

La reconstitution du réseau de sociabilité de Clarissy et de sa seconde épouse Suzanne Berthier a démontré l'existence de liens étroits tissés avec des personnages influents et proches du pouvoir, dont profitent leurs activités commerciales. En effet, Antoine Clarissy doit à plusieurs reprises s'imposer face à la concurrence d'autres céramistes ou verriers qui tentent de l'empêcher de produire ou de vendre ses marchandises. Il rencontre par exemple en 1628 des difficultés avec des potiers de terre parisiens et du Beauvaisis qui vendent leurs productions à la Halle aux blés de Paris, où il avait reçu en 1623 le don d'une place par le roi, en vue de lui permettre de commercialiser ses «vazes, figures et autres ouvrages de terre sigillée ${ }^{33} »$. Il rencontre de la même manière des difficultés avec des verriers parisiens et en particulier Jean
Maréchal, à la tête de la verrerie de Saint-Germain-des-Prés. Fort du monopole qu'il détient sur Paris et trente lieues à la ronde, ce dernier n'hésite pas à s'en prendre à Antoine Clarissy qui, en 1631, ose se lancer dans l'aventure verrière. L'existence éphémère de la verrerie royale d'Antoine Clarissy au domaine du Monceau à Avon de 1641 à 1643 et la réinstallation de sa verrerie dans la capitale qui s'ensuit sont, comme en témoigne un mémoire rédigé par Antoine Clarissy à un moment de détresse financière extrême, la conséquence de ces difficultés ${ }^{34}$. L'adresse de ce mémoire au chancelier de France Pierre Séguier révèle que ce dernier est le protecteur et le soutien d'Antoine Clarissy, ce que confirme par ailleurs la présence du chancelier en qualité de témoin lors du second mariage du céramiste en 1645. Ainsi, les documents d'archives ont permis de restituer à ce personnage toute son importance. Il s'avère en effet qu'Antoine Clarissy est surtout un entrepreneur et un homme d'affaires qui multiplie les associations financières dans des domaines pour lesquels il semblerait n'avoir eu parfois aucune compétence technique spécifique et qui agit grâce et pour le compte de puissants personnages.

Par ailleurs, l'étude d'une centaine d'inventaires après décès a démontré que les terres sigillées issues de la fabrique d'Antoine Clarissy tiennent une place non négligeable parmi les grandes collections d'objets d'art de la première moitié du $\mathrm{XVII}^{\mathrm{e}}$ siècle. Le cardinal de Richelieu et le chancelier Pierre Séguier figurent parmi les clients les plus prestigieux du céramiste et entraînent dans leur sillage la plupart des riches financiers de la capitale qui font à cette même époque bâtir et meubler leurs hôtels particuliers ${ }^{35}$. Les terres sigillées d'Antoine Clarissy sont donc des objets de luxe répondant à un effet de mode. Il est par conséquent surprenant de remarquer que la postérité n'a conservé aucune mémoire de ces objets produits il y a quatre siècles en plein cœur de la capitale. En effet, le terme n'évoque plus de nos jours que de la céramique gallo-romaine. D'abord employé pour désigner exclusivement ce type de céramique décorée de motifs en relief, il désigne aujourd'hui, par extension, toute production de cette époque à vernis rouge ${ }^{36}$. Le mot latin sigillum signifie une petite marque, un sceau ou un cachet, aussi l'hypothèse défendue par Ambroise Milet selon laquelle la terre sigillée produite par Antoine Clarissy correspondrait aux céramiques à glaçure plombifère à décor moulé en relief longtemps considérées comme des suites de Palissy ${ }^{37}$ est-elle cohérente et justifiée. Pour autant, les recherches menées dans les archives parisiennes du XvII ${ }^{\mathrm{e}}$ siècle n'ont pas permis de valider complètement cette hypothèse, car si la description de certains objets répondant à cette dénomination semble pouvoir correspondre, d'autres excluent cette possibilité. C'est par exemple le cas de terres sigillées décrites comme étant de couleur rouge et dorée dans plusieurs inventaires après décès. Ce terme en usage dans les documents de l'époque, qui n'est aujourd'hui plus du tout associé à une production parisienne de la première moitié du XvII siècle, illustre l'imprécision ou l'évolution d'un vocabulaire qui complexifie le travail du chercheur. 


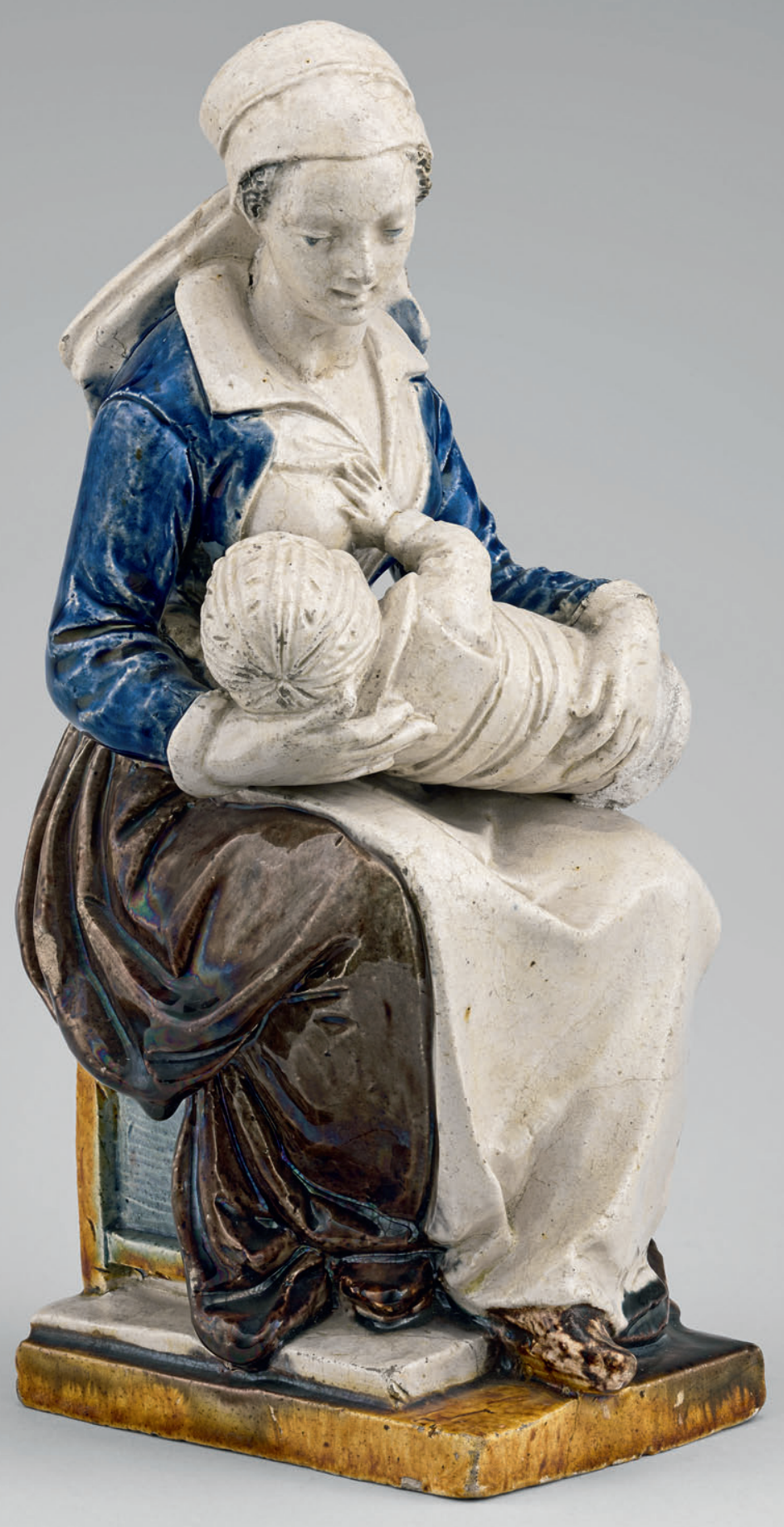




\section{Conclusion}

Qu'il s'agisse des «émailleurs en terre » de Fontainebleau ou de l'ouvrier du roi en terre sigillée Antoine Clarissy, les recherches en archives démontrent aujourd'hui que ces potentiels « suiveurs » ou « continuateurs » que l'on rattachait jusque-là à l'atelier dit «d'Avon ", ont en réalité une activité et des ramifications bien plus importantes dans la capitale. Ces deux pistes de recherche permettent ainsi toutes deux - sans toutefois jamais se croiser - de restituer à Paris son rôle de centre de production et de commercialisation de céramique dans la première moitié du XvII ${ }^{\mathrm{e}}$ siècle. Les archives parisiennes jusqu'à présent moins exploitées par les chercheurs que celles de la cité royale de Fontainebleau recèlent d'informations inédites sur l'art de terre. Ces céramiques à glaçure plombifère et à décor moulé en relief ayant longtemps été interprétées à tort comme l'œuvre ou l'héritage

direct du célèbre potier de la Renaissance, il en résulte l'existence de nombreuses copies et imitations produites au $\mathrm{xIX}^{\mathrm{e}}$ siècle, lorsque l'œuvre de Bernard Palissy a fait l'objet de la plus grande fascination. Originaux et copies se mêlent ainsi aujourd'hui dans la plus grande confusion et l'œil du connaisseur ne permet pas toujours de les distinguer avec certitude. Face aux abyssales lacunes documentaires et à la réévaluation incontournable de l'ensemble des connaissances admises jusqu'ici, l'adoption d'une méthodologie transdisciplinaire s'est imposée et le travail de l'historien et celui du scientifique sont apparus comme parfaitement complémentaires. Le croisement de ces données historiques inédites avec les résultats des analyses physico-chimiques doit ainsi permettre d'acquérir de nouvelles connaissances et révéler peut-être de nouveaux secrets sur ces céramiques fabriquées à la fin du XVI ${ }^{\mathrm{e}}$ siècle et dans la première moitié du XVII ${ }^{\mathrm{e}}$ siècle, et demeurées trop longtemps plongées dans l'ombre de Bernard Palissy.

\section{Notes}

1. Dufaÿ et al., 1987.

2. Perrin, 2001, p. 24-33 ; Bouquillon et al., 2017. Voir l'article de A. Gerbier dans ce volume (p. 16).

3. Barbe, Bouquillon, 2010, p. 30

4. Perrin, 2001, p. 242.

5. Bouquillon et al., 2018 ; Bouquillon et al., 2017.

6. Pour ce qui concerne les fouilles menées en Normandie sur le site dit de la Bosqueterie, voir Bergeret, 2004, p. 23, et pour les fouilles menées en Saintonge sur le site de la Chapelle-des-Pots, voir Henriet, 1988 , p. 27 et les rapports de fouilles conservés au SRA de Charente-Maritime mentionnés dans la bibliographie.

7. Denis-Dupuis, 2018, non publié, vol. II-Annexes, base de données "CEuvres " sur DVD. Herbet, 1897.

8. Dupuis, 2010-2011, non publié ; Dupuis, 2016.

9. Plaziat, 2011 ; Plaziat, 1990, p. 80.

10. Herbet, 1897

11. Voir par exemple : Mc Nab, 1987 ou Rochebrune, 2002.

12. Les lignes qui suivent rendent compte des recherches historiques menées dans le cadre d'une thèse d'histoire moderne réalisée durant trois années et soutenue le 5 juillet 2018 à laquelle nous invitons le lecteur en quête des références des documents d'archives invoqués à se référer : Denis-Dupuis, 2018, thèse non publiée, 2 vol.

13. Héroard, 1989

14. Lhuillier, 1883.

15. Guiffrey, 1872, p. 78

16. Denis-Dupuis, 2018, thèse non publiée, vol. I, p. 282-284.
17. Denis-Dupuis, 2018, thèse non publiée, vol. I, p. 301-344.

18. La foire Saint-Germain est la grande foire annuelle qui a lieu au faubourg Saint-Germain-des-Prés et qui réunit marchands forains et marchands locaux.

Les loges sont les boutiques situées à l'intérieur de la foire.

19. AN, MC/ET/XCVIII/144, 29 novembre 1642 .

20. Perrin, 2001, vol. 2, Annexe A - IX, p. 45 .

21. Guey, 1956 ; AD Seine-Maritime, 2E/1/712, 11 septembre 1608 .

22. AD Seine-Maritime, $2 \mathrm{E} / 1 / 814$, 29 octobre 1621.

23. Denis-Dupuis, 2018, thèse non publiée, vol. I, p. 351-352.

24. Guiffrey, 1872, p. 78-79.

25. Dan, 1642, p. 338-339.

26. Denis-Dupuis, 2018, thèse non publiée, vol. I, p. 381-581. 27. AN, MC/ET/XIX/365, fol. II, 4 janvier 1612 .

28. Bimbenet-Privat, 2002, t. I, p. 103.

29. Bresc-Bautier, Fonkenell, 2016, p. 303 .

30. AN, O/1/1047.

31. La ligne d'eau est la $144^{\mathrm{e}}$ partie du pouce fontainier qui représente un écoulement d'environ 13 litres par minute. 32. BnF, Manuscrits, Fonds Français 16741 , fol. 118 v. Sans date.

33. AN, Z/1f/568, 18 mars 1623. Publiée par Milet, 1876, p. 240-242.

34. BnF, Manuscrits, Fr. 18600 Mémoire intitulé «Sur un privilege d'establir une verrerie » faisant partie des papiers du chancelier Séguier (1539-1654), publié pour la première fois par Paul-Martin
Bondois : Bondois, 1936-1937, p. 58-60. 35. Denis-Dupuis, 2018, thèse

non publiée, vol. I, p. 639-691.

36. Girel, 1996, p. 18.

37. Milet, 1876, p. 238.

\section{Bibliographie}

Barbe F., Bouquillon A., 2010, « À la redécouverte des créations de Bernard Palissy : l'aiguière et le plat à décor de "rustiques figulines" du musée du Louvre ", La Revue des Musées de France, Revue du Louvre, $\mathrm{n}^{\circ}$ 5, p. 24-33.

Bergeret J., 2004, « Les "Suites de Palissy", une vaisselle d'apparat et une vision du monde ", dans Cat. Exp. Lisieux, 2004, Bergeret J. (dir.), Les céramiques du Pré-d'Auge. 800 ans de production [Exp. Lisieux, musée d'Art et d'Histoire de Lisieux, 3 juillet 200430 septembre 2004], musée d'Art et d'Histoire de Lisieux, Lisieux, p. 82-86.

Bimbenet-Privat M., 2002, Les orfèvres et l'orfèvrerie de Paris au XVII siècle, Commission des Travaux Historiques de la Ville de Paris, Paris, 2 vol.

Bondois P.-M., 1936-1937,

«Le développement de l'industrie verrière dans la région parisienne de 1515 à 1665 ", Revue d'histoire économique et sociale, XXIII ${ }^{\mathrm{e}}$ année, $\mathrm{n}^{\circ}$ 1, p. 45-68.

Bouquillon A., Castaing J., Barbe F., CrépinLeblond T., Tilliard L., Paine S. R. Christman B., Heuer A. H., 2018,

"French decorative Ceramic massproduced during and after the $17^{\text {th }}$ Century: Chemical Analyses of the Glazes", Archaeometry, vol. 60, issue 5, p. 946-965. 
Bouquillon A., Castaing J., Barbe F., Paine S. R., Christman B., Crépin-Leblond T., Heuer A.H., 2017, "Lead-glazed Rustiques Figulines (Rustic Ceramics) of Bernard Palissy (1510-90) and his followers", Archaeometry, vol. 59, issue 1, p. 69-83.

Bresc-Bautier G., Fonkenell G. (éd.), 2016, Histoire du Louvre I. Des origines à l'heure napoléonienne, Louvre éditions/Fayard, Paris, vol. 1.

Dan, 1642, Le Trésor des merveilles de la Maison royale de Fontainebleau, Sébastien Cramoisy, Paris.

Dufaÿ B., de Kisch Y., Poulain D., Roumegoux Y., Trombetta P.-J., 1987, "L'atelier parisien de Bernard Palissy ", Revue de l'Art, n ${ }^{\circ} 78$, p. 33-60.

Dupuis J., 2016, « La céramique dite "d'Avon", retour historiographique et mise au jour d'une attribution légendaire ", Revue de l'Art, n 193/20163, p. 27-34.

Girel J., 1996, « La terre sigillée », Revue de la céramique et du verre, $\mathrm{n}^{\circ} 90$, p. 17-26.

Guey F., 1956, «Contribution à l'étude de la Céramique de Rouen ", Revue des Sociétés savantes de Haute-Normandie, $\mathrm{n}^{\circ} 4$, p. 21-33.

Guiffrey J., 1872, « Liste des peintres, sculpteurs, architectes, graveurs et autres artistes de la Maison du roi, de la reine ou des autres princes du sang, pendant les $\mathrm{XVI}^{\mathrm{e}}, \mathrm{XVII}^{\mathrm{e}}$ et XVIII ${ }^{\mathrm{e}}$ siècles ", Nouvelles archives de l'Art français, p. 55-108.

Henriet J. L., 1988, Bulletin de liaison de la SAH Charente-Maritime, $\mathrm{n}^{\circ}$ 15, p. 27.
Herbet F., 1897, « Recherches sur la céramique au XVII ${ }^{\mathrm{e}}$ siècle, les émailleurs sur terre de Fontainebleau ", Annales de la société historique et archéologique du Gâtinais, Maurice Bourges imprimeur, p. 204-245.

Héroard J., 1989, Journal de Jean Héroard, édition établie par Madeleine Foisil, publication du Centre de recherche sur la civilisation de l'Europe moderne, Fayard, Paris.

Lhuillier T., 1883, «Matériaux pour servir à l'histoire de la céramique ", Réunion des sociétés des Beaux-Arts des Départements à la Sorbonne, Paris, Plon, p. 195.

Mc Nab J., 1987, «Palissy et son "école" dans les collections du Metropolitan Museum of Art de New York ", Revue de l'Art, $\mathrm{n}^{\circ} 78$, p. 70-76.

Milet A., 1876, «Antoine Clérissy, ouvrier du roi en terre sigillée (1612-1653). Esquisse sur sa vie et son œuvre ", Nouvelles archives de l'Art français, Paris, p. 230-247.

Perrin I., 2001, Les techniques céramiques de Bernard Palissy, thèse sous la direction de M. Jean Guillaume, soutenue en 1998, université Paris IV-Sorbonne, Anrt, Lille, 2 vol.

Plaziat J.-C., 1990, « Les fossiles du Tertiaire parisien dans l'œuvre scientifique et artistique de Bernard Palissy (15101590), en commémoration du quatrième centenaire de sa mort ", Travaux du Comité français d'histoire de la géologie, COFRHIGEO, $3^{\mathrm{e}}$ série, tome IV, p. 79-84.

Plaziat J.-C., 2011, "Bernard Palissy (15101590) and the French geologists: a critical reappraisal concerning the founding naturalist and his rustic ceramics", Bulletin de la Société géologique de France, t. 182, n 3 , p. 255-267.
Rochebrune M.-L. de, 2002, « L'art de la céramique en France au siècle de Louis XIII ", Un temps d'exubérance, Les arts décoratifs sous Louis XIII et Anne d'Autriche, Galeries nationales du Grand Palais, 9 avril-8 juillet 2002, RMN Éditions, Paris, p. 335-336.

\section{Documents inédits}

Dupuis J., 2010-2011, La céramique d'Avon : limites et paradoxes d'une attribution héritée $d u X X^{e}$ siècle, mémoire de Master I Recherche sous la direction de E. Lurin, université de Paris IV-Sorbonne, $231 \mathrm{p}$.

Denis-Dupuis J., 2018, La céramique à Paris après Bernard Palissy (1590-1650) : auvres, fabricants, collections, thèse de doctorat d'histoire moderne sous la direction de François Pernot, université ParisSeine-Cergy-Pontoise, LabEx Patrima, 2 vol., 923 p.

Henriet, 1983, Fouilles et prospections à la Chapelle-des-Pots : Recherche de l'artisanat céramiste du village.

Henriet, 1984, Les fouilles de la Chapelle-desPots, rapport manuscrit, SRA01263.

Henriet, 1988 et 1989, La Chapelle-des-Pots, fouille de sauvetage urgent, Société d'archéologie de la Charente-Maritime, 3 vol., SRA01269, SRA01270 et SRA01271.

Henriet, 2005, Rapport de fouilles de sauvetage urgent, La Chapelle-des-Pots, propriété M. Krurien, F. Cools, parcelle AL 116, SRA03265. 